\title{
Síndrome Anémico. Carcinoide Gástrico tipo 1 (Papel de la Ultrasonografía endoscópica en el diagnóstico y tratamiento)
}

\author{
Juan Gabriel Córdoba Soriano ${ }^{a}$, Alberto Gato Díez ${ }^{\mathrm{b}}$, Mar Vicente Gutiérrezc, Isabel López Neyra ${ }^{\mathrm{a}}$
}

\begin{abstract}
a Servicio de Cardiología. Complejo Hospitalario Universitario de Albacete (España).

b Servicio de Medicina Interna. Complejo Hospitalario Universitario de Albacete (España).

'Servicio de Aparato Digestivo.

Complejo Hospitalario

Universitario de Albacete

(España).
Correspondencia: Juan Gabriel Córdoba Soriano, Servicio de Cardiología del Hospital General de Albacete,
C/ Hermanos Falcó $n^{\circ} 37$, 02006 - Albacete, España.
Telf.: 620593098,
correo electrónico:
jgCordobas@hotmail.com

Recibido el 7 de mayo de 2010.

Aceptado para su publicación el 30 de julio de 2010.
\end{abstract}

\section{RESUMEN}

Se presenta el caso de un varón de 74 años que ingresa en Medicina Interna por un cuadro de Anemia grave sintomática, alcanzando el diagnóstico de Tumor Neuroendocrino subtipo Carcinoide Gástrico tipo 1 de la Clasificación de Rindi y cols. El papel de la ultrasonografía endoscópica fue crucial para un correcto diagnóstico y tratamiento del paciente. Posteriormente se expone una revisión del estado actual de recomendaciones para un correcto diagnóstico y tratamiento de este tipo de tumores.

Palabras Clave. Anemia Perniciosa, Ultrasonografía Endoscópica, Tumor Carcinoide.

\section{ABSTRACT}

Anemia Syndrome. Type 1 Gastric Carcinoid (Role of endoscopic ultrasound in the diagnosis and treatment)

We report a case of a 74 year old man who was admitted to our Internal Medicine Department for severe symptomatic anemia. He was diagnosed with a type 1 gastric neuroendocrine tumor classified according to Rindi et al, Endoscopic ultrasound was the key for correct diagnosis and patient management. Subsequently, we present a review of the current status of the recommendations for the correct diagnosis and treatment in this type of tumor.

Key words. Anemia, Pernicious, Endosonography, Carcinoid Tumor.

\section{INTRODUCCIÓN}

El Carcinoide Gástrico (CG) es un subtipo de tumor neuroendocrino (TNE) bien diferenciado. La incidencia recogida en la literatura es de 10 casos/100000 hab./año ${ }^{1}$, pero podría ser mayor, según datos de series de autopsias ${ }^{2}$, y su diagnóstico está aumentando debido fundamentalmente a la realización de endoscopias por otros motivos. Este tumor supone cerca de 3 de cada 1.000 neoplasias gástricas ${ }^{1}$, y dentro de los TNE digestivos se recoge una incidencia en la literatura del $3 \%$, aunque en las series más recientes ha aumentado notablemente y varía entre el 11 y el $41 \%^{2}$.

\section{OBSERVACIONES CLÍNICAS}

Se trata de un varón de 74 años, exfumador desde hace más de 20 años y diabético tipo 2. Había sido sometido a una resección de $100 \mathrm{~cm}$ de yeyuno en 1989 por trombosis mesentérica, con trombosis venosa profunda y tromboembolismo pulmonar en el postoperatorio, y a una cistoprostatectomía radical en 2006 por microvejiga secundaria a cistitis ilntersticial e hidronefrosis, que motivó insuficiencia renal crónica con $\mathrm{ClCr}$ de $40 \mathrm{ml} / \mathrm{min}$ y ureterostomía abocada a fosa iliaca derecha. Acudió a nuestro hospital refiriendo un cuadro de 2 meses de evolución de astenia, hiporexia y pérdida de peso de unos 3 $\mathrm{Kg}$ en el último mes, detectándose en la analítica urgente hemoglobina de 6,1 g/dl, con VCM 80,4 fL y CHCM 32,7 g/dl, por lo que se decidió el ingreso del paciente. A la exploración física no se detectaron hallazgos de interés. El estudio analítico de su anemia mostraba: niveles de vitamina B12 descen- 
didos $(124,9 \mathrm{pg} / \mathrm{ml})$ con ácido fólico normal y con perfil ferropénico y unos anticuerpos anti-célula parietal gástrica positivos (1/160). Durante el ingreso se realizó una Gastroscopia que mostró una mucosa intensamente atrófica y telangiectasias en la curvatura mayor medial hacia cara posterior, que fueron coaguladas con argón, apreciándose en la cara antero-medial de cuerpo gástrico una discreta sobreelevación eritematosa de la mucosa, que se biopsió (figura 1), informándose como TNE bien diferenciado que se extendía hasta submucosa. Con estos hallazgos se realizó una determinación de enolasa neuroespecífica en sangre periférica con valores de 23,2 y de 5HIA en orina con valor de 3.1. La determinación de sangre oculta en hece también resultó positiva en dos muestras, realizándose una colonoscopia en la que se detectaron 2 pólipos no resecables endoscópicamente, cuya biopsia fue informada como adenomas vellosos.

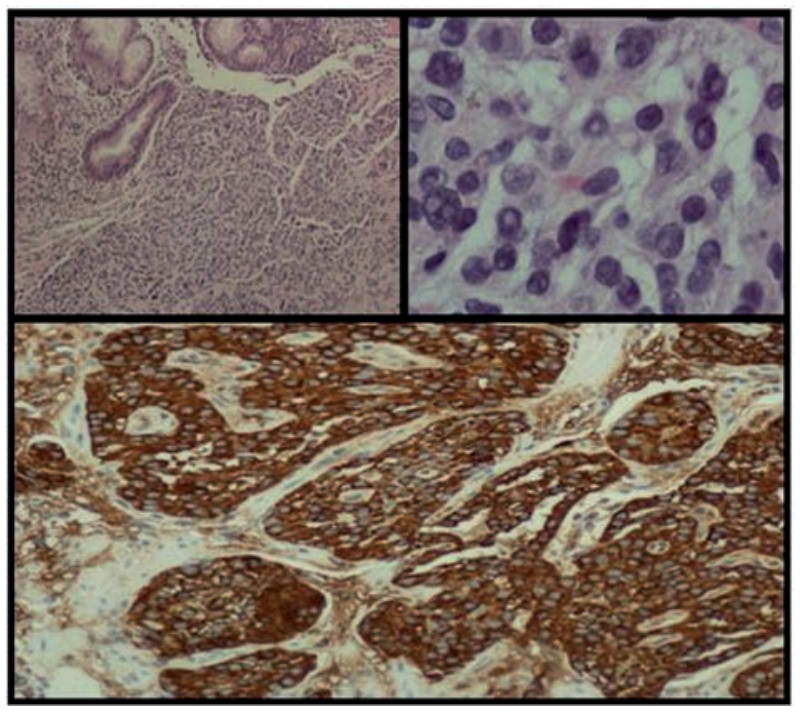

Figura 1. Corte y tinción histológica de la biopsia obtenida por gastroscopia guiada por ecoendoscopia que muestra la presencia de células compatibles con diagnóstico de tumor neuroendocrinológico, con tinción positiva para cromogranina y sinaptofisina.
Una TAC toraco-abdomino-pélvica no reveló la existencia de lesiones a distancia. Con vistas a enfocar el tratamiento definitivo de la lesión gástrica, se realizó una ecoendoscopia gástrica (EEG), que fue informada como lesión de $9 \mathrm{~mm}$ en antro con afectación de las tres capas, que se extendía hasta la muscularis sin sobrepasarla. Llamaba la atención el engrosamiento de la capa muscular. El resto de la pared gástrica era normal (figura 2). Posteriormente un parietograma gástrico por TAC no reveló hallazgos de interés. Una vez completado el estudio y sintetizando las datos encontrados (anemia perniciosa, gastritis crónica atrófica tipo A y carcinoide gástrico), llegamos al diagnóstico de TNE bien diferenciado subtipo carcinoide gástrico tipo 1 de la clasificación de Rindi y cols ${ }^{6}$ (tabla 1). Con ese diagnóstico y cumpliendo criterios para resección endoscópica, se procedió a la misma, sin complicaciones inmediatas y con buen resultado definitivo tras el estudio anatomopatológico de la pieza resecada.

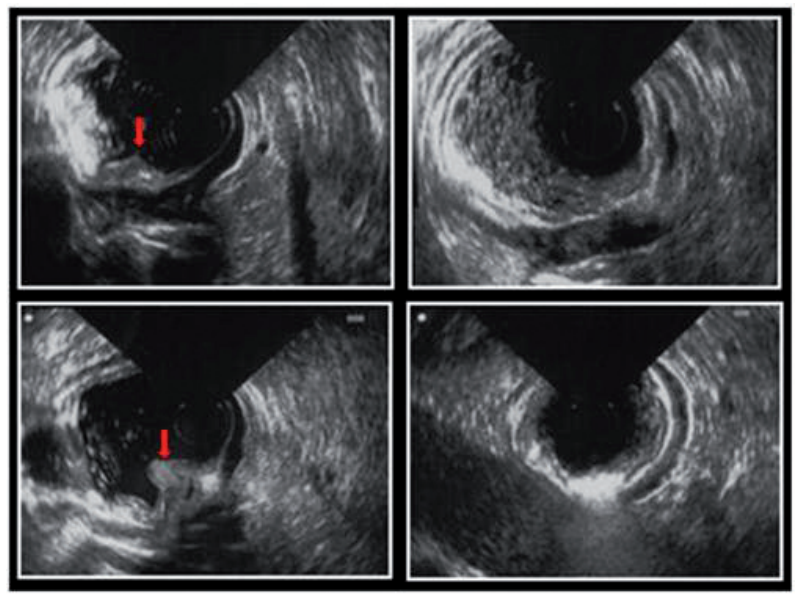

Figura 2. Imagen ecoendoscópica de nuestro paciente en la que puede observarse una sobreelevación de la mucosa con aspecto polipoideo que se extiende hasta la submucosa sin sobrepasarla (flecha roja) y que no era completamente visible en el estudio endoscópico convencional.

\begin{tabular}{l|c}
\hline \multicolumn{1}{c|}{ BIEN DIFERENCIADOS } & MAL DIFERENCIADOS \\
\hline TIPO 1 (80\%) ${ }^{*}$ : Asociado a Gastritis Crónica Atrófica Autoinumne y & \\
Anemia Perniciosa. & Carcinoma Neuroendocrino Gástrico \\
TIPO 2 (6\%)*: Asociado a Síndrome de Zollinger - Ellison y MEN 1 & \\
TIPO $3(\mathbf{1 4 \%})^{*}$ : Esporádico & \\
\hline
\end{tabular}

Tabla 1: Clasificación de los tumores neuroendocrinos gástricos de la OMS basada en la Clasificación de Rindi et al. *Entre paréntesis la frecuencia de presentación de cada tipo. 


\section{COMENTARIOS}

El CG es un tumor infrecuente cuyo tratamiento está clásicamente establecido en la mayoría de los tratados de referencia, pero del que todavía es discutida la modalidad más adecuada, y la forma de caracterización y de guiar este tratamiento más correctamente ${ }^{3,4,5}$. Rindi y cols. establecieron una clasificación de esta neoplasia, aceptada por la mayoría de los autores y recogida en la mayoría de tratados de referencia, en tres tipos, con diferentes manifestaciones clínicas, asociaciones, fisiopatogenia y pronóstico 6,9 .

En los últimos tiempos el tratamiento endoscópico está ganando terreno, con buenos resultados y escasa tasa de complicaciones respecto al tratamiento quirúrgico ${ }^{3,4}$. La complicación más frecuentemente asociada a la técnica es la hemorragia digestiva alta $(4.8-16 \%)$ que según los casos recogidos en la literatura es fácilmente controlable endoscópicamente ${ }^{7}$. Existen varias técnicas de resección endoscópica dependiendo de la protrusión del tumor hacia la luz y del tamaño, debido a la localización fundamentalmente submucosa, en ocasiones poco abordable sin una técnica que lo haga más visible, como la inyección de suero y adrenalina en la submucosa o la ligadura con bandas previa a su resección con asa. Esta indicación está más claramente establecida en tumores menores de $10 \mathrm{~mm}$, de aparición única y con un estudio de extensión negativo, ya que el porcentaje de metástasis hepáticas con estas características suele ser menor del $2 \% 2,3,4,7$. También se sienta una indicación endoscópica en tumores con un tamaño mayor de $10 \mathrm{~mm}(1-2 \mathrm{~cm})$, éstos con un porcentaje de metástasis del $10-15 \%$, que aparecen en pacientes con elevado riesgo quirúrgico y con buenos resultados descritos ${ }^{4,7}$.

A su vez, la ultrasonografía endoscópica (USE) está ganando terreno tanto en la caracterización del tumor con vistas a la adopción de uno u otro tipo de tratamiento, como en una descripción más fidedigna del mismo que ayude tanto al tratamiento quirúrgico como endoscópico. Permite esta última precisar la invasión en profundidad, el tamaño, descubrir lesiones inaparentes en la endoscopia convencional y guiar la resección, así como predecir el comportamiento maligno de las mismas ${ }^{7,8}$. Además la gastroscopia convencional tiene algunos otros inconvenientes, subestimando el tamaño de la lesión en ocasiones y pasando desapercibidas lesiones multicéntricas en otras, debido a que sólo valora el crecimiento intraluminal de las mismas. En nuestro caso permitió definir el tamaño, la invasión en profundidad y el carácter unicéntrico de la lesión, con mayor sensibilidad que el parietograma por TAC, y guiando el tratamiento definitivo del mismo, en un paciente con un riesgo quirúrgico y una comorbilidad ya de por si elevados.

Concluimos, por tanto, que actualmente el tratamiento de los tumores carcinoides gástricos parece beneficiarse claramente de la USE, ya que esta técnica ha demostrado ser la mejor en la caracterización en profundidad de este tipo de lesiones, que presentan un crecimiento intramucoso sin prácticamente expresión intraluminal ${ }^{7}$. Es la más adecuada en la determinación del tamaño y en demostrar la indemnidad de la muscular propia, sentando las bases del tratamiento endoscópico o quirúrgico, ya que estos dos factores son los que se asocian con más fuerza a la posibilidad de aparición de metástasis a distancia ${ }^{7}$. Por tanto, sería conveniente su indicación en todo caso de sospecha de este tipo de tumores.

\section{BIBLIOGRAFÍA}

1. Jensen R T. Tumores endocrinos del tubo digestivo y del páncreas. En: Braunwald E, Fauci AS, Kasper DL et al, editores. Harrison Principios de Medicina Interna. 15a. ed. Madrid: McGraw-Hill; 2001. p. 701-14.

2. Yavasoglu I, Yukselen V, Ozkara E, Ozcara E, Bolaman Z. Treatment of solitary gastric carcinoid tumor by endoscopic polypectomy in a patient with pernicious anemia. World $\mathrm{J}$ Gastroenterol. 2006; 12:4267-9.

3. Fernández-Ruiz M, Rodríguez-Gil Y, Sáenz-López $S$, Guerra-Vales JM, Guillen-Camargo V. Tumor Carcinoide gástrico solitario y anemia perniciosa: tratamiento mediante polipectomía endoscópica. Rev Esp Enferm Dig. 2008; 100(3):186-7

4. Ichikawa J, Tanabe S, Koizumi W, Kida Y, Imaizumi H, Kida $\mathrm{M}$, et al. Endoscopic mucosal resection in the management of gastric carcinoid tumors. Endoscopy. 2003; 35:203- 4.

5. Schindl M, Kaserer K, Niederle B. Treatment of gastric neuroendocrine tumors: The necessity of a type-adapted treatment. Arch Surg. 2001; 136:49-54.

6. Rindi G, Luinetti O, Cornaggia M, Capella C, Solcia E. Three subtypes of gastric argyrophil carcinoid and the gastric neuroendocrine carcinoma: A clinicopathologic study. Gastroenterology. 1993; 104:994-1006.

7. Martínez-Ares D, Souto-Ruzo J, Varas Lorenzo MJ, Espinós Pérez J, Yáñez López J, Abad Belando R et al. Resección Endoscópica asistida por ecoendoscopia de tumores carcinoides del aparato digestivo. Rev Esp Enferm Dig. 2004; 96(12): 847-55.

8. Sjoblom S-M, Sipponen P, Miettinen M, Karonen S-L, Jarvinen HJ. Gastroscopic screening for gastric carcinoids and carcinoma in pernicious anemia. Endoscopy. 1988; 20:52.

9. Kloppel G, Heitz P, Capella C, Solcia E. Pathology and nomenclature of human gastrointestinal neuroendocrine (carcinoid) tumors and related lesions. World J Surg. 1996; 20:13241. 